\title{
THE MANIFOLDS OF LINEAR ELEMENTS OF AN $n$-SPHERE
}

\section{TSAI-HAN KIANG}

1. Introduction. The 3-manifolds of oriented and non-oriented linear elements of closed surfaces have been investigated by Nielsen, ${ }^{1}$ Hotelling, ${ }^{2}$ Threlfall, ${ }^{3}$ van der Waerden and others. ${ }^{4}$ In the present paper we take up the case of the space $M$ of oriented linear elements, and the space $M^{\prime}$ of non-oriented linear elements, of an $n$-sphere, $n \geqq 1$. The chief tools in the present investigation are certain orthogonal transformations ( $\$ \S 3-4)$ and theorems on addition of complexes. ${ }^{5}$ Our success in the determination of certain homology classes $(\$ \$ 7-8$, 14) leads to complete determination of (integral) Betti groups of $M$ and $M^{\prime}$. Our results may be summarized as follows:

(M1) For $n>1, M$ is an orientable $(2 n-1)$-manifold. Its Betti groups, which are not the null groups, are the following: For even $n$, $B^{0}$ and $B^{2 n-1} \approx G_{0}\left(\mathrm{AH}\right.$, p. 556) and $B^{n-1} \approx G_{2}$; for odd $n, B^{0}, B^{2 n-1}$, $B^{n-1}$, and $B^{n} \approx G_{0}$.

(M2) For $n=2, M$ is the projective space. For $n>2$, its fundamental group is the identity.

(M3) For $n=1,3,7, M$ is the topological product of an $n$-sphere and an $(n-1)$-sphere.

$\left(\mathrm{M}^{\prime} 1\right)$ For $n>1, M^{\prime}$ is an orientable or a non-orientable $(2 n-1)$ manifold according as $n$ is even or odd. Its Betti groups, which are not the null, are the following: For even $n, B^{0}$ and $B^{2 n-1} \approx G_{0}$, $B^{n-1} \approx G_{4}$, and $B^{r} \approx G_{2}, r=1,3, \cdots, n-3 ; n+1, n+3, \cdots, 2 n-3$. For odd $n, B^{0}$ and $B^{n} \approx G_{0}$, and $B^{r} \approx G_{2}, r=1,3, \cdots, n-2 ; n+1$, $n+3, \cdots, 2 n-2$.

$\left(\mathrm{M}^{\prime} 2\right)$ For $n=2, M^{\prime}$ is the lens space (Linsenraum) $(4,1) .{ }^{\circ}$ For $n>2$, its fundamental group is the cyclic group of order 2.

Received by the editors October 20, 1944.

${ }^{1} \mathrm{~J}$. Nielsen, Untersuchungen zur Topologie der geschlossen zweiseitigen Flächen, Acta. Math. vol. 50 (1927) pp. 302-306.

${ }^{2} \mathrm{H}$. Hotelling, Three-dimensional manifolds of states of motions, Trans. Amer. Math. Soc. vol. 27 (1925) pp. 329-344; Multiple-sheeted spaces and manifolds of states of motions, ibid. vol. 28 (1926) pp. 479-490.

${ }^{8} \mathrm{~W}$. Threlfall, Räume aus Linienelementen, Jber. Deutschen Math. Verein. vol. 42 (1933) I, pp. 88-110.

4 Solutions of problem 124 by B. L. van der Waerden, H. Kneser, H. Seifert, E. R. van Kampen, and W. Threlfall, Jber. Deutschen Math. Verein. vol. 42 (1933) II, pp. 112-117.

' Alexandroff-Hopf, Topologie I, Berlin (1935), pp. 287-293. This book will be referred to as $\mathrm{AH}$.

' Seifert-Threlfall, Lehrbuch der Topologie, Leipzig, 1934, p. 210. This book will be referred to as ST. 
$\left(\mathrm{M}^{\prime} 3\right)$ For $n=1,3,7, M^{\prime}$ is the topological product of an $n$-sphere and an $(n-1)$-dimensional projective space.

\section{The MANIFold $M$ OF ORIENTED LINEAR ELEMENTS}

2. The representation of $M$. The $n$-sphere, $n \geqq 1$, may be represented in the Euclidean $(n+1)$-space $X$ of the points $\left(x_{0}, x_{1}, \cdots, x_{n}\right)$ by the unit sphere: $x^{\prime} x=x_{0}^{2}+x_{1}^{2}+\cdots+x_{n}^{2}=1$, where $x$ denotes the matrix of one column of the coordinates of the point, and $x^{\prime}$ the transposed of the matrix $x$. Similarly we have $y, y^{\prime}$ and the space $Y$. The space $M$ is then represented by the subspace of the topological product $R=X \times Y$, defined by the equations

$M$

$$
x^{\prime} x=1, \quad x^{\prime} y=0, \quad y^{\prime} y=1 .
$$

Evidently this subspace is a closed $(2 n-1)$-manifold when it is connected (AH, p. 404). Henceforth we shall take this subspace as the space $M$, and speak of the points of $M$ instead of oriented linear elements.

3. The two "halves" of $M$. The decomposition of the $n$-sphere $x^{\prime} x=1$ into the two $n$-cells corresponding to $x_{0} \geqq 0$ and $x_{0} \leqq 0$ gives rise to that of $M$ into the two halves:

$$
\begin{aligned}
& M_{1} \quad x_{0} \geqq 0, \quad x^{\prime} x=1, \quad x^{\prime} y=0, \quad y^{\prime} y=1 \text {; } \\
& M_{2} \quad x_{0} \leqq 0, \quad x^{\prime} x=1, \quad x^{\prime} y=0, \quad y^{\prime} y=1 .
\end{aligned}
$$

Let $e$ denote the matrix of one column of the $n+1$ elements $1,0, \cdots, 0$. The matrix

$$
A_{1}=(x+e)(x+e)^{\prime} /\left(x_{0}+1\right)-I,
$$

where $x^{\prime} x=1, x_{0}+1 \neq 0$, and the matrix

$$
A_{2}=(x-e)(x-e)^{\prime} /\left(x_{0}-1\right)+I,
$$

where $x^{\prime} x=1, x_{0}-1 \neq 0$, are symmetric and orthogonal, and have $x^{\prime}$ and $x$ as their first rows and columns. ${ }^{7}$ Let $u^{(i)}=\left(u_{0}^{(i)}, u_{1}^{(i)}, \ldots, u_{n}^{(i)}\right)$ and $v^{(i)}=\left(v_{0}^{(i)}, v_{1}^{(i)}, \cdots, v_{n}^{(i)}\right)$ denote points in the Euclidean $(n+1)$ spaces $U_{i}$ and $V_{i}$ respectively, $i=1,2$. Form the mappings ${ }^{8}$

$t_{i}$

$$
u^{(i)}=x, \quad v^{(i)}=A_{i} y .
$$

${ }^{7} \mathrm{H}$. W. Turnbull and A. C. Aitken, An introduction to the theory of canonical matrices, London, 1932, Lemma II, p. 104.

8 The author wishes to express his gratitude to his colleague Professor P. L. Hsu for the construction of the matrices $B_{n+1}$ and the mapping $t_{0}$ in $\$ 10$ and for the reference in footnote 7. The construction of $t_{i}$ is then immediate. 
$t_{1}$ and $t_{2}$ are topological mappings of $M_{1}$ and $M_{2}$ respectively on

$K_{1} \quad u_{0}^{(1)} \geqq 0, \quad u^{(1) \prime} u^{(1)}=1, \quad v_{0}^{(1)}=0, \quad v^{(1) \prime} v^{(1)}=1$

in $R_{1}=U_{1} \times V_{1}$ and

$K_{2} \quad u_{0}^{(2)} \leqq 0, \quad u^{(2) \prime} u^{(2)}=1, \quad v_{0}^{(2)}=0, \quad v^{(2) \prime} v^{(2)}=1$

in $R_{2}=U_{2} \times V_{2}$. Let $E_{1}$ be the $n$-cell: $u_{0}^{(1)} \geqq 0, u^{(1)^{\prime}} u^{(1)}=1$ in $U_{1}, E_{2}$ the $n$-cell: $u_{0}^{(2)} \leqq 0, u^{(2) '} u^{(2)}=1$ in $U_{2}$, and $S_{i}$ the $(n-1)$-sphere: $v_{0}^{(t)}=0$, $v^{(i)} v^{(i)}=1$ in $\bar{V}_{i}$. Evidently $K_{i}=E_{i} \times S_{i}$.

4. $M$ as sum. The case $n=1$. Let us denote the subspace: $x_{0}=0$, $x^{\prime} x=1, x^{\prime} y=0, y^{\prime} y=1$ in $R$ by $\operatorname{Bd}\left(M_{1}\right)=\operatorname{Bd}\left(M_{2}\right)$, and the subspace: $u_{0}^{(i)}=0, u^{(i)^{\prime}} u^{(i)}=1$ in $U_{i}$ by $\operatorname{Bd}\left(E_{i}\right)$, and finally set $\operatorname{Bd}\left(K_{i}\right)=\operatorname{Bd}\left(E_{i}\right)$ $\times S_{i}$, the topological product of two $(n-1)$-spheres. Let $P$ be any point of $\operatorname{Bd}\left(M_{i}\right)$. Then $t_{i}(P)=P_{i}$ of $\mathrm{Bd}\left(K_{i}\right)$, and $P_{2}=t_{2} t_{1}^{-1}\left(P_{1}\right)$ is a topological mapping of $\mathrm{Bd}\left(K_{1}\right)$ on $\mathrm{Bd}\left(K_{2}\right)$. Since the symmetric and orthogonal matrix $A_{1}=A_{1}^{\prime}=A_{1}^{-1}$, the topological mapping $t=t_{2} t_{1}^{-1}$ of $\operatorname{Bd}\left(K_{1}\right)$ on $\operatorname{Bd}\left(K_{2}\right)$ is given by $A_{2} A_{1}$, with $x$ in the two factor matrices replaced by $u^{(1)}$. Let $\bar{u}^{(1)}$ denote the matrix of one column of the $n+1$ elements: $0, u_{1}^{(1)}, \cdots, u_{n}^{(2)}$. Then evidently

$t$

$$
u^{(2)}=u^{(1)}, \quad v^{(2)}=\left\{2\left(\bar{u}^{(1)} \bar{u}^{(1) \prime}+e e^{\prime}\right)-I\right\} v^{(1)} .
$$

Through identification of all pairs of points of $\operatorname{Bd}\left(K_{1}\right)$ and $\operatorname{Bd}\left(K_{2}\right)$, corresponding under $t$, there results from $K_{1}$ and $K_{2}$ a sum $K_{1}+K_{2}$. Since $M$ is homeomorphic with $K_{1}+K_{2}$, we shall write $M=K_{1}+K_{2}$.

For $n=1, K_{i}$ consists of two semicircles. By means of $t$, the two end points of each semicircle of $K_{1}$ or $K_{2}$ are identified with the two end points of one and only one semicircle of $K_{2}$ or $K_{1}$ respectively. Hence $M$ is the topological product of a 1 -sphere and a 0 -sphere.

5. The fundamental group. The case $n=2$. For $n>2$, all the fundamental groups of the topological products $K_{i}$ and $\operatorname{Bd}\left(K_{i}\right)$ are the identity (ST, p. 156), and therefore that of $M$ is also the identity (ST, p. 179).

For general $n$, let $\epsilon$ denote the point $(0, \cdots, 0,1)$ in a Euclidean $(n+1)$-space. Let $m_{i}$ and $b_{i}$ denote respectively the $(n-1)$-spheres $\operatorname{Bd}\left(E_{i}\right) \times \epsilon$ and $\epsilon \times S_{i}$ on $\operatorname{Bd}\left(K_{i}\right)$, and be called the meridian and latitude of $\operatorname{Bd}\left(K_{i}\right)$.

In the remaining part of this section we confine ourselves exclusively to the case $n=2$. Denote by $O_{i}$ the point $(\epsilon ; \epsilon)$ of the torus $\operatorname{Bd}\left(K_{i}\right)$ and take it as the initial point of closed oriented curves on $K_{i}$. Obviously $t\left(O_{1}\right)=O_{2}$. The meridian and latitude circles $m_{i}$ and $b_{i}$ will 
be regarded as with definite orientations, fixed as follows. First, take the senses of increasing $\theta$ and $\phi$ in the parametric representations

$m_{1} u_{0}^{(1)}=0, u_{1}^{(1)}=\sin \theta, u_{2}^{(1)}=\cos \theta ; v_{0}^{(1)}=0, v_{1}^{(1)}=0, v_{2}^{(1)}=1$,

$b_{1} \quad u_{0}^{(1)}=0, u_{1}^{(1)}=0, u_{2}^{(1)}=1 ; v_{0}^{(1)}=0, v_{1}^{(1)}=-\sin \phi, v_{2}^{(1)}=\cos \phi$

as the orientations of $m_{i}$ and $b_{i}$ respectively. Now

$\begin{array}{llll} & u_{0}^{(2)}=0, & u_{1}^{(2)}=\sin \theta, & u_{2}^{(2)}=\cos \theta ; \\ t\left(m_{1}\right) & v_{0}^{(2)}=0, & v_{1}^{(2)}=\sin 2 \theta, & v_{2}^{(2)}=\cos 2 \theta, \\ t\left(b_{1}\right) & u_{0}^{(2)}=0, & u_{1}^{(2)}=0, & u_{2}^{(2)}=1 ; \\ & v_{0}^{(2)}=0, & v_{1}^{(2)}=\sin \phi, & v_{2}^{(2)}=\cos \phi .\end{array}$

Let $\left(u^{(i)} ; v^{(i)}\right)$ denote a variable point of a subspace $\Gamma$. We shall call the subspace of $\left(u^{(i)} ; \epsilon\right)$ and that of $\left(\epsilon ; v^{(i)}\right)$ the projections $g_{1}(\Gamma)$ and $g_{2}(\Gamma) . g_{i}$ are evidently continuous mappings of $\Gamma$. The projections $g_{1} t\left(m_{1}\right)$ of $t\left(m_{1}\right)$ and $g_{2} t\left(b_{1}\right)$ of $t\left(b_{1}\right)$ are respectively $m_{2}$ and $b_{2}$. Moreover $g_{1} t$ is a topological mapping of $m_{1}$ on $m_{2}$, and $g_{2} t=t$ a topological mapping of $b_{1}$ on $b_{2}$. Now fix the orientations of $m_{2}$ and $b_{2}$ by demanding that $g_{1} t$ maps the oriented $m_{1}$ on the oriented $m_{2}$ and that $g_{2} t$ maps the oriented $b_{1}$ on the oriented $b_{2}$.

Let $\left[m_{i}\right],\left[b_{i}\right]$ denote the classes of closed oriented curves through $O_{i}$ on $K_{i}$, homotopically deformable into $m_{i}$ and $b_{i}$ when the point $O_{i}$ is kept fixed. The fundamental group of $K_{i}$ is given by the two generators $\left[m_{i}\right],\left[b_{i}\right]$ and the defining relation $\left[m_{i}\right]=1$. From the representation of $t\left(m_{1}\right)$ it is obvious that, when a variable point $P_{1}$ starts from $O_{1}$ and describes the oriented $m_{1}$ once, the point $t\left(P_{1}\right)$ starts from $\mathrm{O}_{2}$ and describes a closed oriented curve on $\mathrm{Bd}\left(K_{2}\right)$ in such a way that its projection $g_{1} t\left(P_{1}\right)$ describes the oriented $m_{2}$ once and its projection $g_{2} t\left(P_{1}\right)$ the oriented $b_{2}$ twice. Furthermore $t$ maps the oriented $b_{1}$ on the oriented $b_{2}$. From the usual consideration of the Euclidean plane as the universal covering of $\mathrm{Bd}\left(K_{2}\right)$, we conclude that in the fundamental group of $K_{2}$,

$$
\left[t\left(m_{1}\right)\right]=\left[m_{2}\right]\left[b_{2}^{2}\right], \quad\left[t\left(b_{1}\right)\right]=\left[b_{2}\right] .
$$

Hence the fundamental group of $M$ is given by the single generator $\left[b_{1}\right]$ and the defining relation $\left[b_{1}^{2}\right]=1$ (ST, pp. 177-178), or is the cyclic group of order 2 .

In fact, $K_{1}, K_{2}$ and the mapping $t$ of $\operatorname{Bd}\left(K_{1}\right)$ on $\operatorname{Bd}\left(K_{2}\right)$ define the Heegaard diagram of the 3-manifold $M$. From the enumeration of all the 3-manifolds whose Heegaard diagrams lie on a torus (ST, 
pp. 210,220$), M$ is the 3 -dimensional projective space. This is a new proof of a well known result. ${ }^{9}$

6. The projections of $t\left(m_{1}\right)$. These projections were used in the preceding section for the determination of the fundamental group of $M$ for $n=2$, and will be investigated here and in the next two sections for the determination in $\$ 9$ of $B^{n-1}(M)$ and $B^{n}(M)$ for $n \geqq 2$. Henceforth suppose always $n \geqq 2$. Now

$\left(t m_{1}\right)$

$$
\begin{aligned}
& u_{0}^{(2)}=0, \quad u_{1}^{(2)}=u_{1}^{(1)}, \cdots, u_{n}^{(2)}=u_{n}^{(1)} \\
& v_{0}^{(2)}=0, \quad v_{1}^{(2)}=2 u_{1}^{(1)} u_{n}^{(1)}, \cdots, v_{n-1}^{(2)}=2 u_{n-1}^{(1)} u_{n}^{(1)},
\end{aligned}
$$

$$
v_{n}^{(2)}=2\left(u_{n}^{(1)}\right)^{2}-1 \text {, }
$$

$$
u_{0}^{(2)}=0, \quad u_{1}^{(2)}=0, \cdots, u_{n-1}^{(2)}=0, \quad u_{n}^{(2)}=1 \text {; }
$$

$$
v_{0}^{(2)}=0, \quad v_{1}^{(2)}=-v_{1}^{(1)}, \cdots, v_{n-1}^{(2)}=-v_{n-1}^{(1)}, \quad v_{n}^{(2)}=v_{n}^{(1)} .
$$

Again obviously $g_{1} t$ is a topological mapping of $m_{1}$ on $m_{2}$ and $g_{2} t=t$ a topological mapping of $b_{1}$ on $b_{2}$. The most important projection $g_{2} t\left(m_{1}\right)$ is given by

$$
\begin{aligned}
u_{0}^{(2)} & =0, \quad u_{1}^{(2)}=0, \cdots, u_{n-1}^{(2)}=0, \quad u_{n}^{(2)}=1, \\
g_{2} t\left(m_{1}\right) \quad v_{0}^{(2)} & =0, \quad v_{1}^{(2)}=2 u_{1}^{(1)} u_{n}^{(1)}, \cdots, v_{n-1}^{(2)}=2 u_{n-1}^{(1)} u_{n}^{(1)}, \\
v_{n}^{(2)} & =2\left(u_{n}^{(1)}\right)^{2}-1 .
\end{aligned}
$$

$g_{2} t$ maps $m_{1}$ continuously in $b_{2}$. It maps all the points $\left(0, u_{1}^{(1)}, \cdots\right.$, $\left.u_{n-1}^{(1)}, 0\right) \times \epsilon$ of $m_{1}$ on the single point $\epsilon \times(0, \cdots, 0,-1)$ of $b_{2}$. When $v_{n}^{(2)} \neq-1$, that is, when $u_{n}^{(1)} \neq 0$, the last $n$ equations above can be solved for $\bar{u}^{(1)}$ in terms of $v_{1}^{(2)}, \cdots, v_{n}^{(2)}$. Hence $g_{2} t$ maps $m_{1}$ on $b_{2}$. Moreover, on the same point $\epsilon \times\left(0, v_{1}^{(2)}, \cdots, v_{n}^{(2)}\right)$ of $b_{2}, g_{2} t$ maps the pair of points $\left(0, \pm u_{1}^{(1)}, \cdots, \pm u_{n}^{(1)}\right) \times \in$ of $m_{1}$, but no other point of $m_{1}$ when $v_{n}^{(2)} \neq-1$. In fact, $g_{2} t$ maps continuously each of the closed halves, $m_{1}^{\prime}$ and $m_{1}^{\prime \prime}$, of $m_{1}$ corresponding to $u_{n}^{(1)} \geqq 0$ and $u_{n}^{(1)} \leqq 0$, on $b_{2}$; and it maps topologically each of the open halves of $m_{1}$, corresponding to $u_{n}^{(1)}>0$ and $u_{n}^{(1)}<0$, on $b_{2}$ minus the point corresponding to $v_{n}^{(2)}=-1$.

Take a simplicial decomposition of the meridian $m_{1}$, symmetric with respect to the center and to the topological product of the hyperplane $u_{n}^{(1)}=0$ and the point $\epsilon$. Take a coherent orientation of this simplicial $m_{1}$. Denote respectively again by $m_{1}, m_{1}^{\prime}, m_{1}^{\prime \prime}$ the $(n-1)$ cycle and the $(n-1)$-complexes, which are the sums of all the

${ }^{9}$ See, for example, Seifert's Solution of problem 124, mentioned in footnote 4; and C. Weber's Solution of problem 84, ibid. pp. 5-6. 
$(n-1)$-simplexes on $m_{1}, m_{1}^{\prime}, m_{1}^{\prime \prime}$, so oriented. By means of the mapping $g_{2} t$, the coherent orientation of $m_{1}^{\prime}$ induces an orientation of $b_{2}$. Take any simplicial decomposition of $b_{2}$ and a coherent orientation determined by this induced orientation. Denote again by $b_{2}$ the $(n-1)$-cycle which is the sum of all the $(n-1)$-simplexes on $b_{2}$ so oriented. Then, from the last paragraph, on $\operatorname{Bd}\left(K_{2}\right)$ the homology class of the singular cycle (ST, p. 97) $g_{2} t\left(m_{1}^{\prime}\right)$ and that of the cycle $b_{2}$ are the same: $\left(g_{2} t\left(m_{1}^{\prime}\right)\right)^{*}=b_{2}^{*}$ on $\operatorname{Bd}\left(K_{2}\right)$.

Now let $Q_{0} Q_{1} \cdots Q_{n-1}$ be an oriented simplex in the coherent orientation of the simplicial $m_{1}$. Let $Q_{i}^{\prime}$ be the diametrically opposite point of $Q_{i}$ on $m_{1}$. Then either the oriented simplex $Q_{0}^{\prime} Q_{1}^{\prime} \cdots Q_{n-1}^{\prime}$ or the oppositely oriented simplex $-Q_{0}^{\prime} Q_{1}^{\prime} \cdots Q_{n-1}^{\prime}$ is in the coherent orientation of $m_{1}$ according as $n$ is even or odd. Hence, on $\operatorname{Bd}\left(K_{2}\right),\left(g_{2} t\left(m_{1}^{\prime \prime}\right)\right)^{*}=b_{2}{ }^{*}$ for even $n$, but $=-b_{2}^{*}$ for odd $n$. Hence, on $\operatorname{Bd}\left(K_{2}\right)$, and therefore also on $K_{2},\left(g_{2} t\left(m_{1}\right)\right)^{*}=2 b_{2}{ }^{*}$ for even $n$, but $=0$ for odd $n$.

7. The homology classes of $t\left(m_{1}\right)$ and $t\left(b_{1}\right)$ on $K_{2}$. To determine the homology class of $t\left(m_{1}\right)$ on $K_{2}$, let us regard the $2 n+2$ equations of $t\left(m_{1}\right)$ in $\$ 6$ as parametric equations of $t\left(m_{1}\right)$ with the $n$ parameters $\bar{u}^{(1)}$, which are the coordinates of a variable point of the $(n-1)$ sphere $S: \bar{u}^{(1)} \bar{u}^{(1)}=1$. Introduce as another parameter the point $\tau$ of the interval $T: 0 \leqq \tau \leqq 1$. After proper simplicial decomposition and coherent orientation of $D=T \times S$, we have as boundary $\operatorname{Bd}(D)$ of $D$ : $\operatorname{Bd}(D)=S_{0}^{\prime}-S_{1}^{\prime}$, where $S_{\tau}^{\prime}$ denotes the topological product of the point $\tau$ and $S$. Now map $D$ continuously in $R_{2}$ by the mapping:

$$
\begin{aligned}
& u_{0}^{(2)}=-\left(1-\tau^{2}\right)^{1 / 2}, u_{1}^{(2)}=\tau u_{1}^{(1)}, \cdots, u_{n}^{(2)}=\tau u_{n}^{(1)} ; \\
& f_{1} \quad v_{0}^{(2)}=0, \quad v_{1}^{(2)}=2 u_{1}^{(1)} u_{n}^{(1)}, \cdots, v_{n-1}^{(2)}=2 u_{n-1}^{(1)} u_{n}^{(1)}, \\
& v_{n}^{(2)}=2\left(u_{n}^{(1)}\right)^{2}-1 .
\end{aligned}
$$

Obviously $f_{1}(D)$ is on $K_{2}$, and $f_{1}=t$ on $S_{1}^{\prime}=m_{1}$. Hence

$$
t\left(m_{1}\right) \sim f_{1}\left(S_{0}^{\prime}\right) \text { on } K_{2} \text {. }
$$

Again after proper simplicial decomposition and coherent orientation of $H=\Sigma \times S$, where $\Sigma$ is the interval: $0 \leqq \sigma \geqq 1$, we have $\operatorname{Bd}(H)=S_{0}^{\prime \prime}-S_{1}^{\prime \prime}$ where $S_{\sigma}^{\prime \prime}$ denotes the topological product of the point $\sigma$ and $S$. Map $H$ in $R_{2}$ by the continuous mapping

$$
\begin{aligned}
& f_{2} u_{0}^{(2)}=-\cos (\sigma \pi / 2), \quad u_{1}^{(2)}=0, \cdots, u_{n-1}^{(2)}=0, \quad u_{n}^{(2)}=\sin (\sigma \pi / 2) \\
& v_{0}^{(2)}=0, \quad v_{1}^{(2)}=2 u_{1}^{(1)} u_{n}^{(1)}, \cdots, v_{n-1}^{(2)}=2 u_{n-1}^{(1)} u_{n}^{(1)}, \quad v_{n}^{(2)}=2\left(u_{n}^{(1)}\right)^{2}-1 .
\end{aligned}
$$


Obviously $f_{2}(H)$ is on $K_{2}, f_{2}=f_{1}$ on $S_{0}^{\prime \prime}=S_{0}^{\prime}$, and $f_{2}\left(S_{1}^{\prime \prime}\right)=g_{2} t\left(m_{1}\right)$. Hence

$$
f_{1}\left(S_{0}^{\prime}\right)=f_{2}\left(S_{0}^{\prime \prime}\right) \sim f_{2}\left(S_{1}^{\prime \prime}\right)=g_{2} t\left(m_{1}\right) \text { on } K_{2} .
$$

This homology together with the preceding one gives

$$
t\left(m_{1}\right) \sim g_{2} t\left(m_{1}\right) \text { on } K_{2} .
$$

From the result at the end of $\$ 6$, we have finally that, on $K_{2},\left(t\left(m_{1}\right)\right)^{*}$ $=2 b_{2}^{*}$ for even $n$, but $=0$ for odd $n$.

Obviously $\left(t\left(b_{1}\right)\right)^{*}=b_{2}^{*}$ on $K_{2}$.

On the sum $K_{1}+K_{2}$, formed by means of the mapping $t, m_{1}^{*}=2 b_{2}^{*}$ for even $n$, but $=0$ for odd $n$, and $b_{1}^{*}=b_{2}^{*}$.

8. The homology classes of $t\left(m_{1}\right)$ and $t\left(b_{1}\right)$ on $\operatorname{Bd}\left(K_{2}\right)$. From Künneth's theorem on the Betti groups of topological product of complexes (AH, p. 308), the Betti groups of $K_{i}$ and $\operatorname{Bd}\left(K_{i}\right)$ together with their bases can be easily determined. All $K_{i}$ and $\operatorname{Bd}\left(K_{i}\right)$ have no torsion coefficients. Their only Betti numbers, which are not zero, are $p^{0}\left(K_{i}\right)=p^{n-1}\left(K_{i}\right)=1$, and $p^{0}\left(\operatorname{Bd}\left(K_{i}\right)\right)=p^{2 n-2}\left(\operatorname{Bd}\left(K_{i}\right)\right)=1$, $p^{n-1}\left(\operatorname{Bd}\left(K_{i}\right)\right)=2$.

Let $m_{i}^{*}$ and $b_{i}^{*}$ denote respectively the homology classes of the $(n-1)$-cycles on $K_{i}$ or $\mathrm{Bd}\left(K_{i}\right)$, which are the sums of all the coherently oriented $(n-1)$-simplexes on certain simplicial decompositions of $m_{i}$ and $b_{i} .{ }^{10}$ Then the $(n-1)$-dimensional homology basis of $K_{i}$ consists of $b_{i}^{*}$ only, while that of $\operatorname{Bd}\left(K_{i}\right)$, of $m_{i}^{*}$ and $b_{i}^{*}$.

The $(n-1)$-dimensional Betti group $B^{n-1}\left(\operatorname{Bd}\left(K_{i}\right)\right)$ is the free Abelian group with the two free generators $m_{i}^{*}$ and $b_{i}^{*}$. The topological mapping $t$ of $\mathrm{Bd}\left(K_{1}\right)$ on $\operatorname{Bd}\left(K_{2}\right)$ induces an isomorphism of $B^{n-1}\left(\mathrm{Bd}\left(K_{1}\right)\right)$ on $B^{n-1}\left(\mathrm{Bd}\left(K_{2}\right)\right)$ (ST, p. 98). This isomorphism must be given by

$$
\left(t\left(m_{1}\right)\right)^{*}=\alpha m_{2}^{*}+\beta b_{2}^{*}, \quad\left(t\left(b_{1}\right)\right)^{*}=\gamma m_{2}^{*}+\delta b_{2}^{*},
$$

on $\operatorname{Bd}\left(K_{2}\right)$, where the coefficients are integers and $\alpha \delta-\beta \gamma= \pm 1 .^{11}$ From the equations of $t\left(b_{1}\right)$ in $\S 6, \gamma=0$; and therefore $\delta= \pm 1$. Hence $\alpha= \pm 1$. Take the simplicial decompositions and coherent orientations of $m_{1}$ and $b_{2}$ as given in $\$ 6$.We can then, and shall, assign coherent orientations to $m_{2}$ and $b_{1}$ such that $\delta=1$ and $\alpha=1$. Hence $\left(t\left(m_{1}\right)\right)^{*}$ $=m_{2}^{*}+\beta b_{2}^{*}$, and $\left(t\left(b_{1}\right)\right)^{*}=b_{2}^{*}$ on $\mathrm{Bd}\left(K_{2}\right)$. The first equation implies

${ }^{10}$ The two interpretations of the symbols $m_{i}^{*}$ and $b_{i}{ }^{*}$ (namely, on $K_{i}$ or on $\operatorname{Bd}\left(K_{i}\right)$ ) will give rise to no confusion, as we shall always state explicitly the complex on which the homology classes are considered.

${ }^{11} \mathrm{~K}$. Reidemeister, Einfiuhrung in die kombinatorische Topologie, Braunschweig, 1932, p. 95. 
$\left(t\left(m_{1}\right)\right)^{*}=\beta b_{2}{ }^{*}$ on $K_{2}$, since $m_{2}^{*}=0$ on $K_{2}$. From the result at the end of $\S 7, \beta=2$ for even $n$, but $=0$ for odd $n$. Hence, on $\operatorname{Bd}\left(K_{2}\right)$, $\left(t\left(m_{1}\right)\right)^{*}=m_{2}^{*}+2 b_{2}^{*}$ for even $n$, but $=m_{2}^{*}$ for odd $n$.

Let $K_{1} \cdot K_{2}$ denote either of the isomorphic sub-complexes of $K_{1}$ and $K_{2}$, which are to be identified in forming the sum $K_{1}+K_{2}$ : $K_{1} \cdot K_{2}=\operatorname{Bd}\left(K_{1}\right)=\operatorname{Bd}\left(K_{2}\right)$. On $K_{1} \cdot K_{2}, m_{1}^{*}=m_{2}{ }^{*}+2 b_{2}{ }^{*}$ for even $n$, but $=m_{2}^{*}$ for odd $n$, and $b_{1}^{*}=b_{2}^{*}$.

9. The Betti groups of $M$. It is obvious that $B^{0}(M) \approx G_{0}$.

Let $N^{r}\left(K_{1} \cdot K_{2}\right)$ be the subgroup of $B^{r}\left(K_{1} \cdot K_{2}\right)$, whose elements are the homology classes of those $r$-cycles, which are null-homologous on both $K_{1}$ and $K_{2} ; S^{r}\left(K_{1}+K_{2}\right)$ the sub-group of $B^{r}\left(K_{1}+K_{2}\right)$, whose elements are the homology classes of those $r$-cycles, each of which is the sum of one $r$-cycle on $K_{1}$ and one $r$-cycle on $K_{2}$. We shall determine as follows $B^{r}(M)$ by means of the theorem:

$$
B^{r}(M)-S^{r}\left(K_{1}+K_{2}\right) \approx N^{r-1}\left(K_{1} \cdot K_{2}\right)
$$

for every $r \geqq 1$ (AH, p. 293).

Let $n$ be even. We say that $S^{n-1}\left(K_{1}+K_{2}\right) \approx G_{2}, N^{2 n-2}\left(K_{1} \cdot K_{2}\right) \approx G_{0}$, and $S^{r}\left(K_{1}+K_{2}\right), N^{r-1}\left(K_{1} \cdot K_{2}\right)$ are the null for all other values of $r \geqq 1$. Let us prove first for example $S^{n-1}\left(K_{1}+K_{2}\right) \approx G_{2}$. Any $(n-1)$ cycle on $K_{i}$ is homologous on $K_{i}$ to an integral multiple of $b_{i}$. Hence any $(n-1)$-cycle on $K_{1}+K_{2}$, which is a sum of one $(n-1)$-cycle on $K_{1}$ and one $(n-1)$-cycle on $K_{2}$, is homologous on $K_{1}+K_{2}$ to a linear combination of $b_{1}$ and $b_{2}$ with integral coefficients. From the results in $\S 7,2 b_{2}^{*}=0$ and $b_{1}^{*}=b_{2}^{*}$ on $K_{1}+K_{2}$. Moreover, $b_{1}^{*} \neq 0$ on $K_{1}+K_{2}$. For, if otherwise, let $C$ be a complex on $K_{1}+K_{2}$, such that $\operatorname{Bd}(C)=b_{1}$. Let $C=C_{1}+C_{2}$, where $C_{i}$ is on $K_{i}$. Then $b_{1}-\operatorname{Bd}\left(C_{1}\right)=\operatorname{Bd}\left(C_{2}\right)$. This shows that the right member would be on $K_{2}$, while the left member would be on $K_{1}$. Hence $\operatorname{Bd}\left(C_{2}\right)$ and $b_{1}-\operatorname{Bd}\left(C_{1}\right)$, and therefore $\operatorname{Bd}\left(C_{1}\right)$, would be all on $K_{1} \cdot K_{2}$. Being on $K_{1} \cdot K_{2}$ and null-homologous on $K_{i}$, $\operatorname{Bd}\left(C_{i}\right)$ would be homologous on $K_{1} \cdot K_{2}$ to an integral multiple of $m_{i}$, say $\lambda_{i} m_{i}$. Hence $b_{1}^{*}=\lambda_{1} m_{1}^{*}+\lambda_{2} m_{2}^{*}$ on $K_{1} \cdot K_{2}$, which is impossible from §8. Hence $b_{1}^{*} \neq 0$ on $K_{1}+K_{2}$, and $S^{n-1}\left(K_{1}+K_{2}\right) \approx G_{2}$. Next $N^{n-1}\left(K_{1} \cdot K_{2}\right) \approx G_{1}$ follows from $\S 8$. The other isomorphisms stated are obvious, and we shall omit the proof.

Hence for every $r \geqq 1$, at least one of the two groups $S^{r}\left(K_{1}+K_{2}\right)$ and $N^{r-1}\left(K_{1} \cdot K_{2}\right)$ is the null, and, from the theorem stated above, $B^{r}(M)$ is isomorphic with the other group. Consequently $B^{n-1}(M)$ $\approx G_{2}, B^{2 n-1}(M) \approx G_{0}$, and $B^{r}(M) \approx G_{1}$ for all other values of $r \geqq 1$.

Similarly, when $n$ is odd, $S^{n-1}\left(K_{1}+K_{2}\right), N^{n-1}\left(K_{1} \cdot K_{2}\right), N^{2 n-2}\left(K_{1} \cdot K_{2}\right)$ all $\approx G_{0}$, and $S^{r}\left(K_{1}+K_{2}\right), N^{r-1}\left(K_{1} \cdot K_{2}\right) \approx G_{1}$ for all other values 
of $r \geqq 1$. Consequently $B^{n-1}(M), B^{n}(M)$ and $B^{2 n-1}(M)$ all $\approx G_{0}$, and $B^{r}(M) \approx G_{1}$ for all other values of $r \geqq 1$.

Thus the proof of the statements in (M1) and (M2) in $\$ 1$ is completed.

10. The cases $n=1,3,7 .^{8}$ Let

$$
B_{8}=\left(\begin{array}{rrrrrrrr}
x_{0} & x_{1} & x_{2} & x_{3} & x_{4} & x_{5} & x_{6} & x_{7} \\
x_{1} & -x_{0} & x_{3} & -x_{2} & x_{5} & -x_{4} & -x_{7} & x_{6} \\
x_{2} & -x_{3} & -x_{0} & x_{1} & x_{6} & x_{7} & -x_{4} & -x_{5} \\
x_{3} & x_{2} & -x_{1} & -x_{0} & x_{7} & -x_{6} & x_{5} & -x_{4} \\
x_{4} & -x_{5} & -x_{6} & -x_{7} & -x_{0} & x_{1} & x_{2} & x_{3} \\
x_{5} & x_{4} & -x_{7} & x_{6} & -x_{1} & -x_{0} & -x_{3} & x_{2} \\
x_{6} & x_{7} & x_{4} & -x_{5} & -x_{2} & x_{3} & -x_{0} & -x_{1} \\
x_{7} & -x_{6} & x_{5} & x_{4} & -x_{3} & -x_{2} & x_{1} & -x_{0}
\end{array}\right) .
$$

Let $B_{4}$ be the four-rowed square matrix at the upper left corner of $B_{8}$, and $B_{2}$ the two-rowed square matrix at the upper left of $B_{4}$. For $n=1,3,7, B_{n+1}$ has $x^{\prime}$ and $x$ as the first row and column, and is orthogonal. The mapping

$$
u=x, \quad v=B_{n+1} y
$$

is a topological mapping of the whole $M$, for $n=1,3,7$, on

$$
u^{\prime} u=1, \quad v_{0}=0, \quad v^{\prime} v=1,
$$

the topological product of an $n$-sphere and an $(n-1)$-sphere. Hence we have our statement (M3) in $\$ 1$.

\section{THE MANIFOLD $M^{\prime}$ OF NON-ORIENTED LINEAR ELEMENTS}

11. $M^{\prime}$ as sum. The cases $n=1,3,7$. By the symbol $(x ; y) \rightleftarrows(x ;-y)$ we mean that the two points $(x ; y)$ and $(x ;-y)$ are to be identified. The space $M^{\prime}$ of non-oriented linear elements of an $n$-sphere is then represented by the equations of $M$ and the additional condition $(x ; y) \rightleftarrows(x ;-y)$. In this sense we shall say for simplicity that $M^{\prime}$ is $M$ after diametrical identification $(x ; y) \rightleftarrows(x ;-y)$. From the symmetry of $x$ and $y$ in the equations of $M, M^{\prime}$ is homeomorphic with the space given by the equations of $M$ and the additional condition $(x ; y) \rightleftarrows(-x ; y)$, and therefore is also the space of oriented linear elements of an $n$-dimensional projective space. $M^{\prime}$ is obviously a closed $(2 n-1)$-manifold if it is connected.

Let $M^{\prime}$ be decomposed also into two halves $M_{i}^{\prime}, i=1,2$, namely 
the $M_{i}$ after diametrical identification $(x ; y) \rightleftarrows(x ;-y)$. From the nature of the equations of the topological mapping $t_{i}$ on $M_{i}, t_{i}$ carries $(x ; y)$ and $\left(x^{*} ; y^{*}\right)$ respectively into $\left(u^{(i)} ; v^{(i)}\right)$ and $\left(u^{(i)} ;-v^{(i)}\right)$ when and only when $\left(x^{*} ; y^{*}\right)=(x ;-y)$. Hence $t_{i}$ is a topological mapping of $M_{i}^{\prime}$ on $K_{i}^{\prime}$, namely the $K_{i}$ after diametrical identification $\left(u^{(i)} ; v^{(i)}\right) \rightleftarrows\left(u^{(i)} ;-v^{(i)}\right)$. Let $\pi_{i}$ be the $S_{i}$ in $V_{i}(\S 3)$ after diametrical identification $v^{(i)} \rightleftarrows-v^{(i)}$, and $\operatorname{Bd}\left(M_{i}^{\prime}\right), \operatorname{Bd}\left(K_{i}^{\prime}\right)$ the $\operatorname{Bd}\left(M_{i}\right), \operatorname{Bd}\left(K_{i}\right)$ in $\$ 4$ after diametrical identifications. Then we have here $K_{i}^{\prime}=E_{i} \times \pi_{i}$, $\operatorname{Bd}\left(M_{1}^{\prime}\right)=\operatorname{Bd}\left(M_{2}^{\prime}\right)$ and $\operatorname{Bd}\left(K_{i}^{\prime}\right)=\operatorname{Bd}\left(E_{i}\right) \times \pi_{i}$. The mapping $t$ is a topological mapping of $\operatorname{Bd}\left(K_{1}^{\prime}\right)$ on $\operatorname{Bd}\left(K_{2}^{\prime}\right)$. Thus, in the sense of homeomorphism, $M^{\prime}=K_{1}^{\prime}+K_{2}^{\prime}$, the sum being defined by means of $t$.

In the particular cases $n=1,3,7$, from the mapping $t_{0}$ in $\$ 10$, topological on the whole $M^{\prime}$, we conclude that $M^{\prime}$ is the topological product of an $(n-1)$-sphere and an $(n-1)$-dimensional projective space.

12. The fundamental group. The case $n=2$. Let us denote by $\pi_{i}^{r}$ the $r$-dimensional projective space: $v_{0}^{(i)}=0, v^{(i)} v^{(i)}=1, v_{r+2}^{(i)}=\ldots$ $=v_{n}^{(i)}=0, v^{(i)} \rightleftarrows-v^{(i)}$. In particular, $\pi_{i}^{n-1}$ is our $\pi_{i}$ previously defined. Let $\sigma_{i}^{r}=\epsilon \times \pi_{i}^{r}$. For $n>2$, the fundamental group of $K_{i}^{\prime}$ or $\operatorname{Bd}\left(K_{i}^{\prime}\right)$ is the cyclic group of order 2 with the class of the oriented $\sigma_{i}^{1}$ as the generator (ST, p. 156). From the equations of $t\left(b_{1}\right)$ in $\S 6$, we know that $t$ maps the oriented $\sigma_{1}^{1}$ into properly oriented $\sigma_{2}^{1}$. Therefore the fundamental group of $M^{\prime}$ is the cyclic group of order 2 (ST, pp. 177178).

When $n=2, \operatorname{Bd}\left(K_{i}^{\prime}\right)$ is the torus. From $\S 5$ together with a necessary change of notations, we know that the fundamental group of $K_{i}^{\prime}$ is given by two generators $\left[m_{i}\right]$ and $\left[\sigma_{i}\right]$ and the defining relation $\left[m_{i}\right]=1$, and that

$$
\left(\left[t\left(m_{1}\right)\right]\right)=\left[m_{2}\right]\left(\left[\sigma_{2}^{1}\right]\right)^{4}, \quad\left(\left[t\left(\sigma_{1}^{1}\right)\right]\right)=\left[\sigma_{2}^{1}\right] .
$$

Hence the fundamental group of $M^{\prime}$ is the cyclic group of order 4 (ST, pp. 177-178), in agreement with Threlfall's result, ${ }^{8}$ and $M^{\prime}$ is the Linsenraum $(4,1)$ (ST, pp. 219-220, 210, 215).

13. The Betti groups of $K_{i}^{\prime}$ and $\operatorname{Bd}\left(K_{i}^{\prime}\right)$. Henceforth we always assume $n \geqq 2$. For simplicity, let our $\sigma_{i}^{n-1}$ previously defined be denoted by $\sigma_{i}$.

The Betti groups of the factors of $K_{i}^{\prime}$ and $\operatorname{Bd}\left(K_{i}^{\prime}\right)$ together with their bases are well known. The Betti groups of $K_{i}^{\prime}$ and $\operatorname{Bd}\left(K_{i}^{\prime}\right)$ together with their bases can be easily determined from Künneth's theorem. The Betti groups $B^{0}$ of $K_{i}^{\prime}$ and $\operatorname{Bd}\left(K_{i}^{\prime}\right)$ are all $\approx G_{0}$, and the others are listed as follows. 
When $n$ is even, $B^{r}\left(K_{i}^{\prime}\right) \approx G_{2}, r=1,3, \cdots, n-3$, with the basis $\left(\sigma_{i}^{r}\right)^{*}$, $B^{n-1}\left(K_{i}^{\prime}\right) \approx G_{0}$ with the basis $\sigma_{i}^{*}, B^{r}\left(\operatorname{Bd}\left(K_{i}^{\prime}\right)\right)$ and $B^{n-1+r}\left(\operatorname{Bd}\left(K_{i}^{\prime}\right)\right)$ $\approx G_{2}$ with the respective bases $\left(\sigma_{i}^{r}\right)^{*}$ and $\left(\operatorname{Bd}\left(E_{i}\right) \times \pi_{i}^{r}\right)^{*}, B^{n-1}\left(\operatorname{Bd}\left(K_{i}^{\prime}\right)\right)$ $\approx G_{0}+G_{0}$ with the basis $m_{i}^{*}$ and $\sigma_{i}^{*}, B^{2 n-2}\left(\operatorname{Bd}\left(K_{i}^{\prime}\right)\right) \approx G_{0}$ with the basis $\left(\operatorname{Bd}\left(E_{i}\right) \times \pi_{i}\right)^{*}$, and all the rest are the null.

When $n$ is odd, $B^{r}\left(K_{i}^{\prime}\right) \approx G_{2}, r=1,3, \cdots, n-2$, with the basis $\left(\sigma_{i}^{r}\right)^{*}, B^{r}\left(\operatorname{Bd}\left(K_{i}^{\prime}\right)\right)$ and $B^{n-1+r}\left(\operatorname{Bd}\left(K_{i}^{\prime}\right)\right) \approx G_{2}$ with the respective bases $\left(\sigma_{i}^{r}\right)^{*}$ and $\left(\operatorname{Bd}\left(E_{i}\right) \times \pi_{i}^{r}\right)^{*}, B^{n-1}\left(\operatorname{Bd}\left(K_{i}^{\prime}\right)\right) \approx G_{0}$ with the basis $m_{i}^{*}$, and all the rest are the null.

14. The homology classes of $t\left(m_{1}\right), t\left(\sigma_{1}\right)$ and $t\left(\operatorname{Bd}\left(E_{1}\right) \times \pi_{1}^{r}\right)$ on $\operatorname{Bd}\left(K_{2}^{\prime}\right)$. We shall assume as in $\$ 6$ that, for even $n, \sigma_{i}$ has a simplicial decomposition and a proper orientation. For odd $n$, the projective space $\sigma_{i}$ is non-orientable and there is no longer any non-null (integral) $(n-1)$-cycle on it. We assume only that $\sigma_{i}$ in this case has a simplicial decomposition and that all its $(n-1)$-simplexes are oriented so that $t$ carries the integral complex $\sigma_{1}$ into the integral complex $\sigma_{2}$. Regarding $\sigma_{i}$ as $b_{i}$ after diametrical identification, we can apply to $M^{\prime}$ the discussions in $\$ \$ 6-8$ with only slight modification in arguments and with necessary change of notations. From these sections we have evidently the following results: On $K_{i}^{\prime},\left(t\left(m_{1}\right)\right)^{*}=4 \sigma_{2}^{*}$ for even $n$, but $=0$ for odd $n$, and $\left(t\left(\sigma_{1}\right)\right)^{*}=\sigma_{2}^{*}$ for even $n$. Hence on the sum $M^{\prime}=K_{1}^{\prime}+K_{2}^{\prime}, m_{1}^{*}=4 \sigma_{2}^{*}$ for even $n$, but $=0$ for odd $n$, and $\sigma_{1}^{*}=\sigma_{2}^{*}$ for even $n$. On $\operatorname{Bd}\left(K_{2}^{\prime}\right),\left(t\left(m_{1}\right)\right)^{*}=m_{2}^{*}+4 \sigma_{2}^{*}$ for even $n$, but $=m_{2}^{*}$ for odd $n$, and $\left(t\left(\sigma_{1}\right)\right)^{*}=\sigma_{2}^{*}$ for even $n$. Hence on $K_{1}^{\prime} \cdot K_{2}^{\prime}$, $m_{1}^{*}=m_{2}^{*}+4 \sigma_{2}^{*}$ for even $n$, but $=m_{2}^{*}$ for odd $n$, and $\sigma_{1}^{*}=\sigma_{2}^{*}$ for even $n$.

Suppose that $\sigma_{i}^{r}$ and $\operatorname{Bd}\left(E_{i}\right) \times \pi_{i}^{r}, r=1,3, \cdots, n-3, n-1$ for even $n$ and $r=1,3, \cdots, n-2$ for odd $n$, has simplicial decompositions and proper coherent orientations. From the nature of the equations of $t\left(b_{1}\right)$ in $\$ 6$,

$$
\left(t\left(\sigma_{1}^{r}\right)\right)^{*}= \pm\left(\sigma_{2}^{r}\right)^{*}
$$

and since $t$ is a topological mapping of $\mathrm{Bd}\left(K_{1}^{\prime}\right)$ on $\mathrm{Bd}\left(K_{2}^{\prime}\right)$, it induces an isomorphism of $B^{n-1+r}\left(\operatorname{Bd}\left(K_{1}^{\prime}\right)\right)$ on $B^{n-1+r}\left(\operatorname{Bd}\left(K_{2}^{\prime}\right)\right)$ :

$$
\left(t\left(\mathrm{Bd}\left(E_{1}\right) \times \pi_{1}^{r}\right)\right)^{*}= \pm\left(\mathrm{Bd}\left(E_{2}\right) \times \pi_{2}^{r}\right)^{*} \text {. }
$$

We can, and shall, assume that the coherent orientations of $\sigma_{i}^{r}$ and $\operatorname{Bd}\left(E_{i}\right) \times \pi_{i}^{r}$ have been so chosen that the signs in the above equations are all positive. Hence on $K_{1}^{\prime} \cdot K_{2}^{\prime},\left(\sigma_{1}^{r}\right)^{*}=\left(\sigma_{2}^{r}\right)^{*},\left(\operatorname{Bd}\left(E_{1}\right) \times \pi_{1}^{r}\right)^{*}$ $=\left(\operatorname{Bd}\left(E_{2}\right) \times \pi_{2}^{r}\right)^{*}$.

15. The Betti groups of $M^{\prime}$. Obviously $B^{0}\left(M^{\prime}\right)$ is the infinite cyclic group, and $M^{\prime}$ is connected. 
Let $n$ be even. We say that $S^{r}\left(K_{1}^{\prime}+K_{2}^{\prime}\right) \approx G_{2}, r=1,3, \cdots, n-3$, $S^{n-1}\left(K_{1}^{\prime}+K_{2}^{\prime}\right) \approx G_{4}, N^{r-1}\left(K_{1}^{\prime} \cdot K_{2}^{\prime}\right) \approx G_{2}, r=n+1, n+3, \cdots, 2 n-3$, $N^{2 n-2}\left(K_{1}^{\prime} \cdot K_{2}^{\prime}\right) \approx G_{0}$, and $S^{r}\left(K_{1}^{\prime}+K_{2}^{\prime}\right), N^{r-1}\left(K_{1}^{\prime} \cdot K_{2}^{\prime}\right)$ are the null for all other values of $r \geqq 1$. The proof of these isomorphisms is based on the results in $\$ \$ 13-14$ and an argument similar to that used in $\S 9$. Then, as in $\S 9$, we have $B^{r}\left(M^{\prime}\right) \approx G_{2}, r=1,3, \cdots, n-3 ; n+1, n+3$, $\cdots, 2 n-3, B^{n-1}\left(M^{\prime}\right) \approx G_{4}, B^{2 n-1}\left(M^{\prime}\right) \approx G_{0}$, and $B^{r}\left(M^{\prime}\right)$ are the null for all other values of $r \geqq 1 . M^{\prime}$ is therefore orientable, when $n$ is even.

Similarly, when $n$ is odd, $S^{r}\left(K_{1}^{\prime}+K_{2}^{\prime}\right) \approx G_{2}, r=1,3, \cdots, n-2$, $N^{r-1}\left(K_{1}^{\prime} \cdot K_{2}^{\prime}\right) \approx G_{2}, r=n+1, n+3, \cdots, 2 n-2, N^{n-1}\left(K_{1}^{\prime} \cdot K_{2}^{\prime}\right) \approx G_{0}$, and $S^{r}\left(K_{1}^{\prime}+K_{2}^{\prime}\right), N^{r-1}\left(K_{1}^{\prime} \cdot K_{2}^{\prime}\right)$ are the null for all other values of $r \geqq 1$. Hence $B^{r}\left(M^{\prime}\right) \approx G_{2}, r=1,3, \cdots, n-2 ; n+1, n+3, \cdots, 2 n-2$, and $B^{n}\left(M^{\prime}\right) \approx G_{0}$, and $B^{r}\left(M^{\prime}\right)$ are the null for all other values of $r \geqq 1$. $M^{\prime}$ is therefore non-orientable when $n$ is odd.

The National University of Peking and Academia Sinica 